\title{
Duodenal Stump Leak Post Curative Gastrectomy for Early Gastric Cancer Patient Treated with Endoscopic Stent which Complicated by Migration and Perforation
}

\section{Bandar Ali*, Jin Won Lee and Kyo Young Song}

Department of Surgery, Minimal Access and Robotic Surgery Center, Seoul St. Mary's Hospital, College of Medicine, The Catholic University of Korea, Seoul, Korea

\begin{abstract}
Purpose: This report was designed to review the critical complication following gastrectomy that can cause devastating problems in duodenal stump leakage patients. Although a non-operative approach might effectively manage such a complication without compromising patients' clinical conditions, it is not always uneventful.

Presentation of case: A 47 year old male with not known comorbidities diagnosed to have early gastric cancer and underwent a laparoscopic distal gastrectomy with Billroth-1 reconstruction. On postoperative day 9, the patient visited emergency department with a new history of persistent nausea, fatigue, and severe abdominal pain. His diagnostic workup revealed evidence of anastomotic site leak, which was initially treated by an endoscopic stent. Unfortunately, the clinical condition of the patient worsens and complicated by stent migration. One day later, the patient underwent laparoscopic exploration; drainage and gastrojejunostomy were performed uneventfully.

Discussion: The leaking duodenal stump following gastric resection presents a definite and critical problem in gastric surgery and especially with gastric cancer. An anastomotic leak is one of the most serious complications following gastric surgery cases. It is associated with intra-abdominal sepsis which can result in significant morbidity and mortality. Early appropriate management should be categorized and management strategy should be implemented according to the size of the leak, extent of the abscess, and clinical status of the patient of the patient. It can be planned with a less invasive procedure. This is no always an uneventful procedure and close observation is mandatory.

Conclusion: Anastomosis or duodenal stump leaks must be diagnosed as early as possible, and treated appropriately with non-operative methods if possible. Non-operative methods not always uneventful intervention and complication of each procedure should be always expected. Regardless of the operative technique the key to appropriate treatment stabilizes the patient, repair the anastomosis leak site, and adequate drainage.
\end{abstract}

Keywords: Gastric cancer; Duodenal stump leak; Endoscopic stent; Surgical intervention; Complication

\section{Introduction}

Management of all complications following any intervention is very important and should be basic principles. When gastric cancer patient develops a duodenal stump leak post gastrectomy, the situation will be crucial due to the aggressiveness of gastric cancer, suspected association of malnutrition and immunocompromised patients. It can result in significant morbidity and could even lead to mortality, as the associated intra-abdominal sepsis carries a high mortality rate. Early recognition and ideal treatment modality are necessary to achieve less morbidity and mortality; however, the treatment may be difficult and varies according to the clinical status of the patient, underlying diseases, the extent and grade of the leak.

In some recent reports, the leak rate of $1-3 \%$ and mortality can reach up to rate $50 \%[1,2]$. In non-complicated duodenal stump leak, the drainage can be done by simple interventional radiological methods, i.e., percutaneous tube duodenostomy (TD), or endoscopic stent or surgical intervention. Close observation post-drainage procedures are very important as the patient condition may be worsened due to duodenal stump dehiscence, so early surgical treatment is necessary [3].

In this paper, we review a case of a dual complication of duodenal stump leakage after laparoscopic distal gastrectomy and Billroth [1] reconstruction in a patient with early gastric cancer stage were located in the distal gastric antrum. Initially, it was treated by endoscopic stent which was, unfortunately, complicated by migration and perforation.
This case is interesting not only because of the delayed presentation of anastomosis site leak but also because it underlines the need for attention of less invasive procedure which can worsen the situation and add more complication to the primary complication needed for treatment. It also extends to the gastrointestinal surgeons to be familiar with the surgical techniques that can provide the ability to properly manage postoperative complication

\section{Case Presentation}

A 47 year old male patient otherwise healthy, who was referred to our surgical clinic after incidental endoscopic finding during routine screening check-up. The patient's history wise only remarkable for alcohol drinking occasionally, smoker, but not taking any medication and no family history for any malignancy.

Esophagogastroduodenoscopy (EGD), which identified two

${ }^{*}$ Corresponding author: Bandar Ali, Department of Surgery, Minimal Access and Robotic Surgery Center, Seoul St. Mary's Hospital, College of Medicine, The Catholic University of Korea, Seoul, Korea505 Banpo-dong, Seocho-gu, Seoul, 137-701, Korea, Tel: +82-2-2258-6365; E-mail: biaa1003@yahoo.com

Received March 25, 2016; Accepted May 20, 2016; Published May 27, 2016

Citation: Ali B, Lee JW, Song KY (2016) Duodenal Stump Leak Post Curative Gastrectomy for Early Gastric Cancer Patient Treated with Endoscopic Stent which Complicated by Migration and Perforation. J Oncol Transl Res 2: 107.

Copyright: (c) 2016 Ali B, et al. This is an open-access article distributed under the terms of the Creative Commons Attribution License, which permits unrestricted use, distribution, and reproduction in any medium, provided the original author and source are credited. 
Citation: Ali B, Lee JW, Song KY (2016) Duodenal Stump Leak Post Curative Gastrectomy for Early Gastric Cancer Patient Treated with Endoscopic Stent which Complicated by Migration and Perforation. J Oncol Transl Res 2: 107.

Page 2 of 4

adjacent lesions anterior wall of the proximal antrum of the stomach. Endoscopic biopsy reported as a moderately differentiated adenocarcinoma. Computed Tomography (CT) of the abdomen was done and showed collapsed stomach, however, no gross lesion can be seen. An about $3 \times 2 \mathrm{~cm}$ sized lobulated mass lesion in the hepatic angle most likely hemangioma as known early gastric cancer metastasis is less. No evidence of local or systemic metastasis. Bone scan was negative study for bone metastasis

The patient underwent uneventful curative laparoscopic distal gastrectomy, D1+ lymph node dissection with Billroth1 reconstruction and discharged home in good condition in post op D7 (Figure 1).

2 days post discharge, which was D9 post operation, the patient visited the emergency department with a complaint of RUQ abdominal pain, Nausea but no vomiting. General appearance, acute ill-looking appearance, mildly dehydrated but not cyanotic neither icteric. Vital signs were, BP 127/55 mm Hg - HR 72/min - RR 22/min - BT 36.8, Mental status, Alert and fully conscious. Upon abdominal examination, the abdomen was mildly distended, voluntary guarding in the right upper abdomen but no sign for diffuse peritonitis. Normal activity for bowel sound and wound dressing site was dry and clean.

Patient resuscitated with IVF, analgesic, antibiotic and prophylactic anticoagulation. Laboratory workup showed mildly elevated leukocyte with left shift neutrophilia. Chemistry and coagulation profile was within normal range. ESR was elevated $(54 \mathrm{~mm} / \mathrm{h})$, CRP (4.05). Then the patient shifted for CT abdomen, which showed Postoperative changes including a complicated fluid collection in the perihepatic space and porta hepatis. Most likely leakage at the gastroduodenostomy site.

The gastroenterologist was contacted for endoscopic stent insertion which was done uneventfully. After stent insertion patient shifted to high dependency unit (HDU) for close observation, vital signs monitoring. The patient kept NPO on IVF and antibiotics started.

On D2 post stent insertion, patient's parameters changed dramatically again, i.e., fever/chill (38.8/+), B.P (100/60), generalized abdominal tenderness, rebound tenderness and Muscle guarding (Figures 2-5).

The patient had been taken to the theatre for laparoscopic exploration and the findings were marked adhesion at RUQ area, Phlegmon formation between anastomosis, liver, GB, and T-colon, Pus drainage (later culture showed coagulase negative staphylococcus), Terminal ileitis and appendix was grossly normal. The impression of anastomosis leakage persistent due to inappropriate stent coverage (partially) or migration. Vigorous irrigation and drainage procedure done. Reconstruction performed by gastrojejuonostomy.

Intra-operative image showing the phlegmon and severe adhesion around duodenal stump, gallbladder and liver

On post-opD1 $v / s$ 105/65-75-20-38.2.JPLt 120cc-serousanguinous/ Rt 150cc-serous still NPO and antibiotics (Figures 6-8).

The patient general condition improved day by day, vital signs stabilized and remained in the normal limits. Abdominal drains got clear more and more. Oral diet resumed on day 4 post op. by sips of water and progressed slowly as tolerated.

On day 5 post op. upper GI series performed and resulted as negative for leakage. Patient discharged home in day 11 post op in good condition (Figures 9 and 10).

\section{Discussion}

Early identification and proper treatment strategy for duodenal stump leak are very important. Surgeons should be aware of the many options and different procedures that have been described for dealing with such complication. The appropriate treatment methods were determined accordingly to the each patient's clinical situation. Surgery for prevention of further leakage was considered in cases of

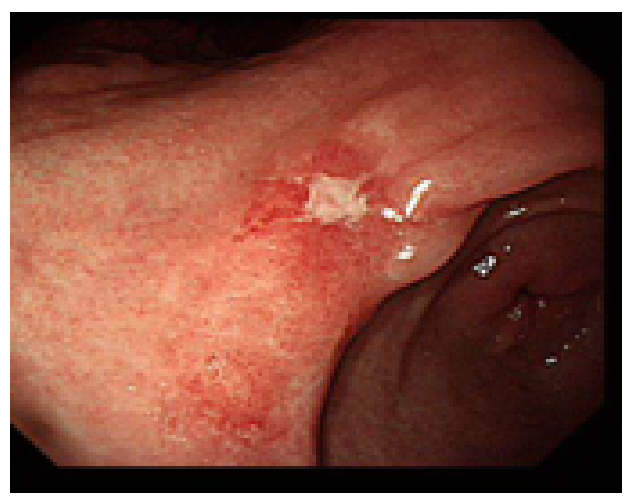

Figure 1: Gross type: Two adjacent lesions, Bormann 3, anterior wall of proximal antrum, $1.2 \times 1.3 \mathrm{~cm}$ ulcerative lesion $\rightarrow$ indigo carmine apply, Biopsy G1-G2.

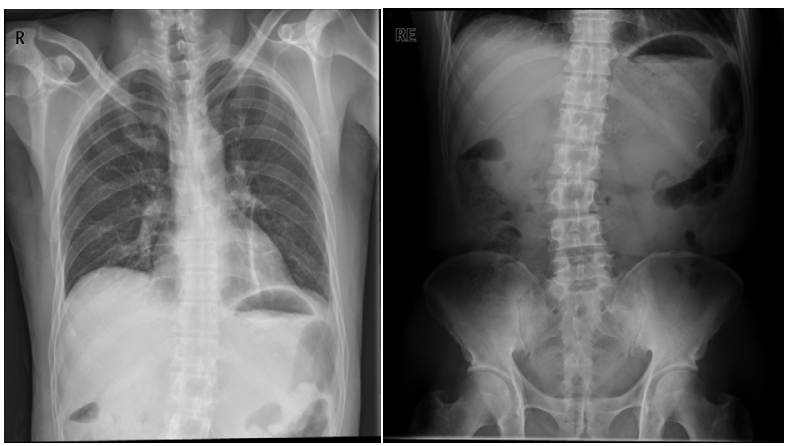

Figure 2: CHEST PA shows a long vertical curved thick linear opacity in LLL behind the heart, suggesting a sub segmental atelectasis. A shorter and thinner vertical sub segmental atelectasis is also seen in RLL. Comparison with prior study reveals disappearance of mild bilateral pleural effusion. Post op. AXR showed a moderate amount of the gastric contents.

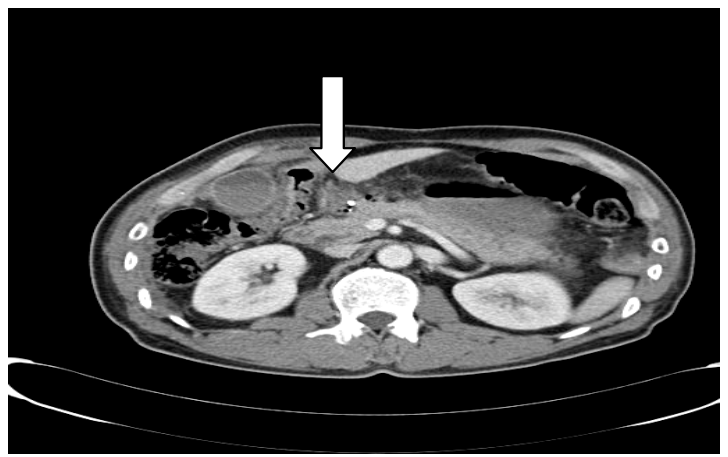

Figure 3: CT abdomen showed post-operative changes including complicated fluid collection in the perihepatic space and porta hepatis. Most likely Leakage at the gastro-duodenostomy site. 
Citation: Ali B, Lee JW, Song KY (2016) Duodenal Stump Leak Post Curative Gastrectomy for Early Gastric Cancer Patient Treated with Endoscopic Stent which Complicated by Migration and Perforation. J Oncol Transl Res 2: 107.

Page 3 of 4
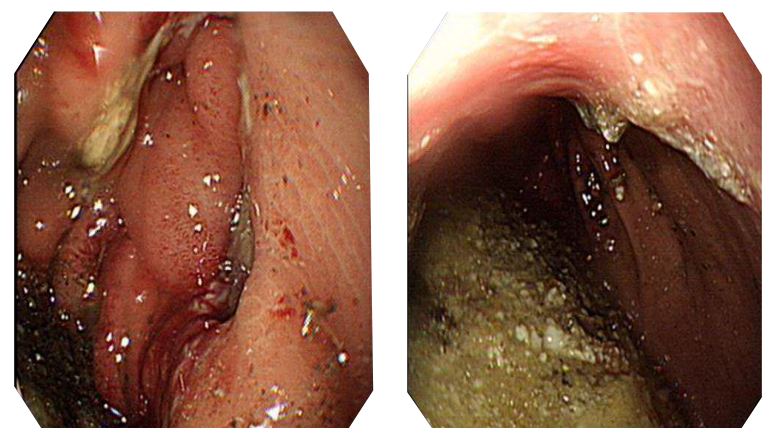

Figure 4: a \& b: B-I STG state large amount of food material edematous narrowing and angulation of anastomosis site suspicious mucosal defect covered with whitish plaque, anastomosis site, GC/PW side.

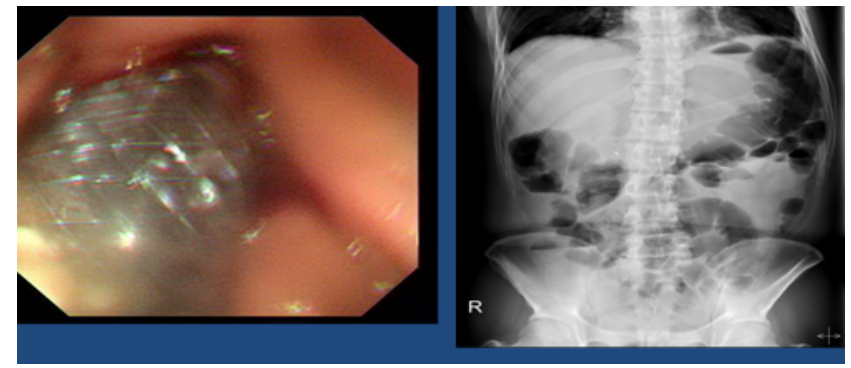

Figure 5: Gastric sleeve stent. B-I STG state much food material still remained in stomach. Anastomosis site edema, Niti-S STENT fully covered, $18 \mathrm{~mm}, 8$ $\mathrm{cm}$ stent insertion (Lot No.PST1080F).

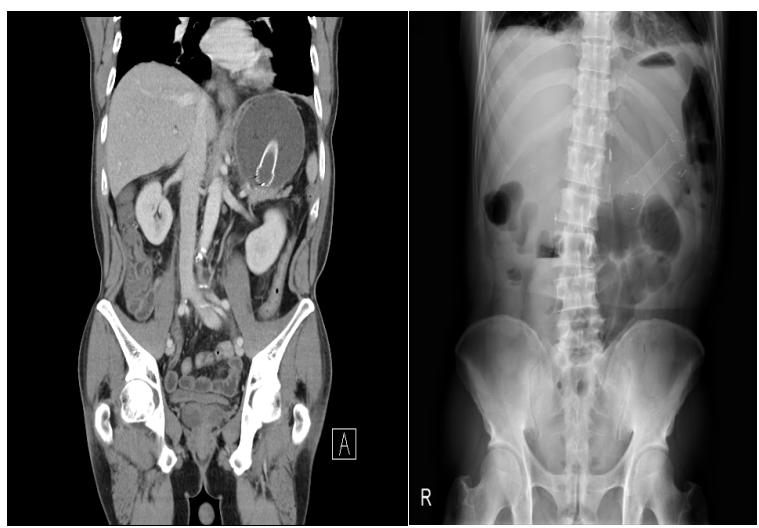

Figure 6: CT abdomen (coronal view) S/P Billroth I subtotal gastrectomy. The Stent in distended stomach is still noted and gastric outlet obstruction. R/O cholecystitis, probably due to partial biliary obstruction with mild IHD dilatation.

diffuse peritonitis, intra-abdominal haemorrhage, and major wound disruption. The non-invasive or nonsurgical management will be important also to support early recovery and promote healing. This category includes parenteral or enteral nutrition (nasojejunal feeding), high dose proton pump inhibitors, and broad spectrum antibiotics and anti-inflammatory medications.One of the interesting theories we would like to raise in this case is the initial cause of duodenal stump leak in the first surgery done for this patient. Although in the era of advancing new technologies, i.e., electrocautery devices to facilitate and simplify the surgical procedures, the safety of this technology should be considered and much revision must be taken.
Endoscopic treatment for the leak is a great option and less invasive than surgery. With advancing in this tool, the stents were introduced and was in the early 1990s. It was mainly for permanent treatment of tumor stenosis in cancer patients with an advanced disease. Although there is no clear data supporting the duration of stenting and removal, it is widely acceptable practice for removal as soon as possible when the patient clinical condition improves [2]. The stent has many varieties in according to its size, manufactured material, and shape. One of these called a self-expanding metallic stent (SEMS). It should remain inside the gut to overcome the leak and achieve healing [3,4]. Fully covered self-expanding metal stent (SEMS) placement has been successfully described for the treatment of malignant and benign conditions. It is not without complication and the reported rate of complication was ranging between 23\%-29\% [5,6]. Whenever surgical complication encountered, the procedure should be kept recorded to be available for review and lessons must be shared to minimize these complications.

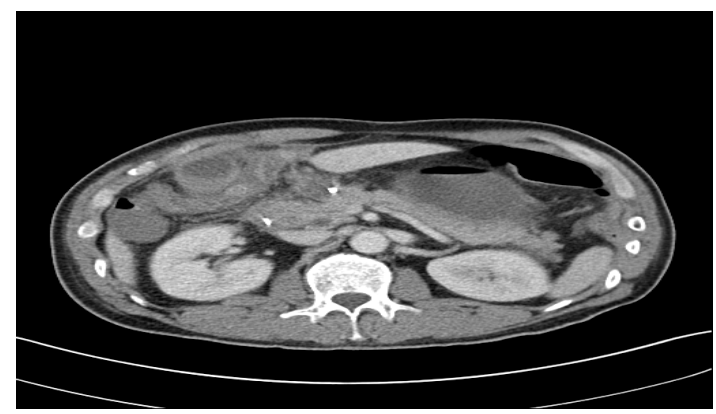

Figure 7: CT abdomen (cross-section) S/P Billroth I subtotal gastrectomy. The Stent in the distended stomach is still noted and gastric outlet obstruction. R/O cholecystitis, probably due to partial biliary obstruction with mild IHD dilatation.

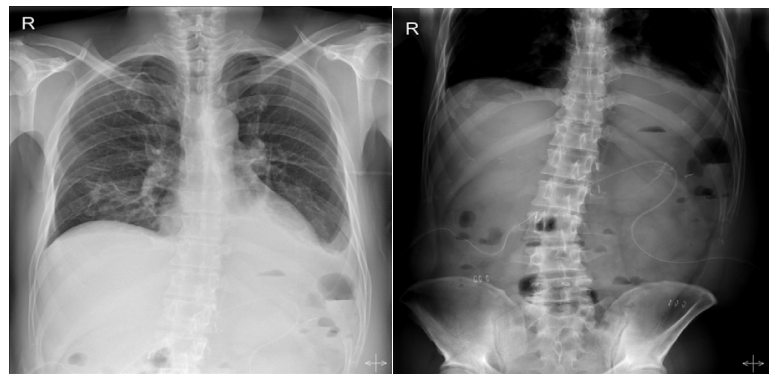

Figure 8: Post-operative AXR with abdominal drain in situ.

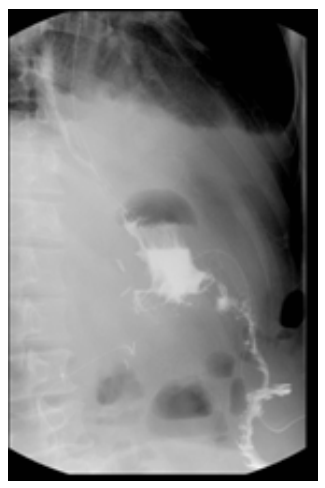

Figure 9: UpperGI series performed and resulted as negative for leakage. 

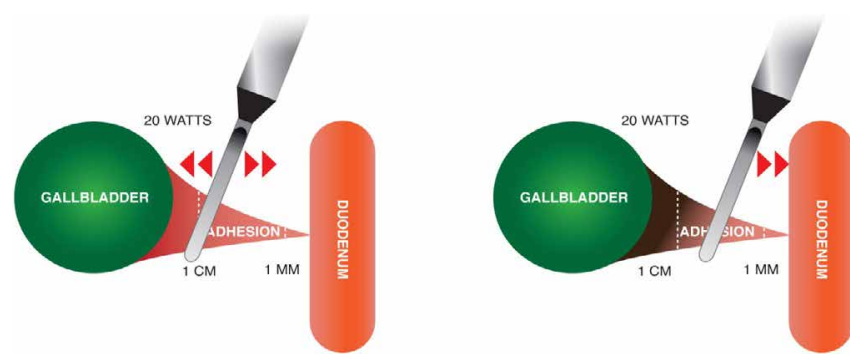

Figure 10: Current concentration theory and tissue damage.

The outcomes in the duodenal stump leaks can be varied and dependable on the learning curve of the endoscopist. Some hospitals also on a routine practice fluoroscopic exam in every patient on the first three days post-surgery with water soluble gastrograffin or barium sulfate.

Tsuei et al. [1] have proposed a classification of leaks based on time of appearance after surgery, clinical severity, and location of leaks. Leaks have been classified based on the period they appear as early, between first and fourth-day post op, intermediate, between the fifth and ninth day after surgery, and late, appearing after day ten. Type I or subclinical are those that appear as a localized leak, without spillage, with few clinical manifestations and are easy to treat medically. Type 2 is those with dissemination into the abdominal or even pleural cavity [7].

Many theories came up to explain the mechanism of duodenal stump leak. But most authors suggested that the major risk factor for gastric leaks is not stapled line dehiscence but is due to the compromised blood supply in the gastric wall next to the stapled line [1]. Classic ischemic fistulas tend to appear between 5 and 6 days after surgery when the wall-healing process is between the inflammation and fibrotic phase. Mechanical fistulas are usually discovered earlier, within the first 2 days after surgery [6].

The clinical picture of patients with gastric leak varies from the completely asymptomatic patient to sepsis, multi-organ failure, and death. The most common signs and symptoms were fever, epigastric pain, tachycardia, and leukocytosis [7]. A methylene blue test, gastrografin study, and computerized axial tomography can aid and help in formatting the diagnosis.

One of the important theories to be focused on in this paper, which is known as Current Concentration theory. It is related to the thermal cautery, which refers to a process in which a direct or alternating current is passed through a resistant metal wire electrode. The heated electrode is then applied to living tissue to achieve hemostasis, but timing and magnitude of heat will be the causing of damages [8].

Many points should be kept in mind during usage of this technique and must consider the different histological configuration of the tissue needed to be treated, the area and depth of destruction desired, possible complications, and capabilities of the different electrocautery devices. A common principle of all electrosurgical procedures is to use the least amount of power possible to achieve the desired effect, limiting damage to the adjacent tissue.

Tissue damage is proportional to the power applied to the area of contact. It has three elements. The function of the variable resistance of tissues, the function of tissue desiccation and the function of current density.
Remote (distal) injuries can be explained by this theory .The problem of unintended tissue damage has often been attributed to current's purported tendency to concentrate around, and follow, certain structures such as blood vessels, causing un-physiologic heating and subsequent damage. It must be realized that current will tend to distribute through tissue in such a manner as to minimize the potential difference between the electrodes. Remote injuries can be explained by this theory.

To make electro-surgery usage simple, it must be clear that the effects obtained are the result of thermal energy released by the generator. This thermal energy may be derived from an external source and transmitted to tissue by the conductance of the diathermy [9].

Neither excessive use nor under use of high frequencies is desirable in the surgical procedures. Misuse of this tool can lead to catastrophic surgical complications. From tissue necrosis to non-healing wounds all are undesirable collateral damages. The active tip may be visualized but the electrode shaft may be lying on viscera outside the view of the scope. In the absence of surveillance, it is possible for a tiny defect in the insulation to deliver (unobserved) 100\% of the current to nontarget tissue $[9,10]$.

The fully developed art of electrosurgery is reflected by the surgeon's ability to manipulate fine electrodes with appropriate wattage without causing further tissue damage.

\section{Conclusion}

Although the revolution in the minimally invasive procedures in the medical fields is promising, it is not without serious side effects or collateral damages. Radiological or endoscopic approach seems to be a feasible option as a primary care modality for patients with postoperative gastric leak although with some variable and acceptable complications rate which should be awarded by the clinician.

Early recognition of complication after any kind of intervention is very important for better outcome. Regarding the electrosurgical devices, the surgeon should be mindful of the risks involved and be proactive to ensure the patient's safety during its usage.

\section{References}

1. Tsuei BJ, Schwartz RW (2004) Management of the difficult duodenum. Curr Surg 61: 166-171.

2. Donatelli G, Dhumane P, Perretta S, Dallemagne B, Vix M (2012) Endoscopic placement of fully covered self-expanding metal stents for management of postoperative foregut leaks. J Minim Access Surg 8: 118-124.

3. Siersema PD (2005) Treatment of esophageal perforations and anastomotic leaks: The endoscopist is stepping into the arena. Gastrointest Endosc 61: 897-900.

4. White RE, Mungatana C, Topazian M (2003) Expandable stents for iatrogenic perforation of esophageal malignancies. J Gastrointest Surg 7: 715-719.

5. Leers JM, Vivaldi C, Schäfer H, Bludau M, Brabender J, et al. (2009) Endoscopic therapy for esophageal perforation or anastomotic leak with a self-expandable metallic stent. Surg Endosc 23: 2258-2262.

6. Fukumoto R, Orlina J, McGinty J, Teixeira J (2007) Use of polyflex stents in the treatment of acute esophageal and gastric leaks after bariatric surgery. Surg Obes Relat Dis 3: 68-72.

7. Salinas A, Baptista A, Santiago E, Antor M, Salinas H (2006) Self-expandable metal stents to treat gastric leaks. Surg Obes Relat Dis 2: 570-572.

8. Pollock SV, Bolognia JL, Jorizzo JL, Rapini RP (2008) Electrosurgery Dermatology. Mosby Elsevier: Ch140.

9. Davison J, Zamah N (2008) Glob libr women's med.

10. Larson BB, Foreman RC (1951) Syndrome of the leaking duodenal stump. AMA Arch Surg 63: 480-485. 